\title{
On Non-Poissonian Voronoi Tessellations
}

\author{
M. Ferraro ${ }^{1} \&$ L. Zaninetti ${ }^{1}$ \\ ${ }^{1}$ Dipartimento di Fisica, Via Pietro Giuria 1, 10125, Turin, Italy \\ Correspondence: L. Zaninetti, Dipartimento di Fisica, Via Pietro Giuria 1, 10125, Turin, Italy. E-mail: za- \\ ninetti@ph.unito.it
}

\author{
Received: October 15, 2015 Accepted: October 25, 2015 Online Published: November 19, 2015 \\ doi:10.5539/apr.v7n6p108 URL: http://dx.doi.org/10.5539/apr.v7n6p108
}

\begin{abstract}
The Voronoi tessellation is the partition of space for a given seeds pattern and the result of the partition depends completely on the type of given pattern "random", Poisson-Voronoi tessellations (PVT), or "non-random", Non Poisson-Voronoi tessellations. In this note we shall consider properties of Voronoi tessellations with centers generated by Sobol quasi random sequences which produce a more ordered disposition of the centers with respect to the PVT case. A probability density function for volumes of these Sobol Voronoi tessellations (SVT) will be proposed and compared with results of numerical simulations. An application will be presented concerning the local structure of gas $\left(\mathrm{CO}_{2}\right)$ in the liquid-gas coexistence phase. Furthermore a probability distribution will be computed for the length of chords resulting from the intersections of random lines with a three-dimensional SVT. The agreement of the analytical formula with the results from a computer simulation will be also investigated. Finally a new type of Voronoi tessellation based on adjustable positions of seeds has been introduced which generalizes both PVT and SVT cases.
\end{abstract}

Keywords: 07.05.Tp, Computer modeling and simulation, 89.75.Da, Scaling phenomena in complex systems

\section{Introduction}

Three-dimensional Voronoi tessellations produce a random partition of the space which have found applications ranging from geology, Blower, Keating, Mader, and Phillips (2002) and molecular biology (Poupon, 2004; Dupuis et al., 2011) to numerical computing (for a review see Du \& Wang, 2005 and references therein), and chemistry (Jedlovszky, Medvedev, \& Mezei, 2004; Idrissi, Vyalov, Kiselev, Fedorov, \& Jedlovszky, 2011).

In most studies Voronoi tessellations have been considered in which the positions of the centers are randomly distributed, giving rise to the so called Poisson-Voronoi tessellations (PVT) (Okabe, Boots, Sugihara, \& Chiu, 2000) even though examples of non Poissonian Voronoi Tessellation can be found in the literature (Heinrich \& Schiile, 1995; Chiu \& Quine, 2011; González \& Einstein, 2011). Non uniform distributions of the centers can be of interest to model regular physical configurations; we shall consider here the properties of Voronoi tessellations whose center are generated by Sobol quasi random sequences (Sobol, 1967; Bratley \& Fox, 1988).

A probability density function (PDF) for volumes of these Sobol Voronoi tessellations (SVT) will be proposed and compared with the results of numerical simulations.

In section 4 SVT and PVT will be used in an application concerning the local structure of gas $\left(\mathrm{CO}_{2}\right)$ in the liquidgas coexistence phase.

In addition, we shall consider the relations between these three-dimensional structures and their lower dimensional sections; in particular we shall study chord length distributions resulting from the random intersection of SVT with straight lines. In case of PVT probability density functions of chords can be derived rigorously (Muche \& Stoyan, 1992; Muche, 2010; ), see also Okabe, Boots, Sugihara, and Chiu (2000); here we shall present an empirical method to calculate a PDF of chord lengths in the case of SVT.

Finally Voronoi tessellations derived by perturbating the positions of points lying on a regular lattice will be considered. 


\section{Probability Density Functions}

In the case of one-dimensional PVT, with average linear density $\lambda$, it has been proved (Kiang, 1966) that the distribution of the lengths of the segments has PDF

$$
p(l)=4 \lambda^{2} l \exp (-2 \lambda l) .
$$

At the present time there are no analytical formulae for the area's and volume's distribution and we limited ourself to explore the available conjectures. Numerical experiments have shown that for PVT an approximate solution can be obtained via a 3 parameters generalized Gamma distribution, that in case of a variable $x$ is

$$
G(x ; a, b, c)=\frac{a b^{\frac{c}{a}} x^{c-1} \mathrm{e}^{-b x^{a}}}{\Gamma\left(\frac{c}{a}\right)},
$$

see (Hinde \& Miles, 1980; Tanemura, 2003; Khodabin \& Ahmadabadi, 2010; Lazar, Mason, MacPherson, \& Srolovitz, 2013). The main moments of $G(x ; a, b, c)$ are:

$$
\begin{gathered}
\langle x\rangle=\frac{b^{-a^{-1}} \Gamma\left(\frac{1+c}{a}\right)}{\Gamma\left(\frac{c}{a}\right)}, \\
\sigma^{2}=\frac{b^{-2 a^{-1}}\left(\Gamma\left(\frac{c+2}{a}\right) \Gamma\left(\frac{c}{a}\right)-\left(\Gamma\left(\frac{1+c}{a}\right)\right)^{2}\right)}{\left(\Gamma\left(\frac{c}{a}\right)\right)^{2}},
\end{gathered}
$$

the skewness $\gamma$ is

$$
\gamma=\frac{\Gamma\left(\frac{3+c}{a}\right)\left(\Gamma\left(\frac{c}{a}\right)\right)^{2}-3 \Gamma\left(\frac{1+c}{a}\right) \Gamma\left(\frac{c+2}{a}\right) \Gamma\left(\frac{c}{a}\right)+2\left(\Gamma\left(\frac{1+c}{a}\right)\right)^{3}}{\left(\Gamma\left(\frac{c+2}{a}\right) \Gamma\left(\frac{c}{a}\right)-\left(\Gamma\left(\frac{1+c}{a}\right)\right)^{2}\right)^{3 / 2}},
$$

and the kurtosis $k$

$$
k=\frac{N_{1}+N_{2}}{D},
$$

with

$$
\begin{array}{lc}
N_{1}= & \left(\Gamma\left(\frac{c}{a}\right)\right)^{3} \Gamma\left(\frac{4+c}{a}\right)-4 \Gamma\left(\frac{1+c}{a}\right)\left(\Gamma\left(\frac{c}{a}\right)\right)^{2} \Gamma\left(\frac{3+c}{a}\right) \\
N_{2}= & 6\left(\Gamma\left(\frac{1+c}{a}\right)\right)^{2} \Gamma\left(\frac{c}{a}\right) \Gamma\left(\frac{c+2}{a}\right)-3\left(\Gamma\left(\frac{1+c}{a}\right)\right)^{4} \\
D= & \left(\Gamma\left(\frac{c+2}{a}\right) \Gamma\left(\frac{c}{a}\right)-\left(\Gamma\left(\frac{1+c}{a}\right)\right)^{2}\right)^{2} .
\end{array}
$$

Usually, instead of $v$ the reduced variable $x=v /\langle v\rangle$ is used (Tanemura, 2005): fitting procedures carried out in (Tanemura, 2005) give best parameters values $a=1.16788, b=4.04039, c=4.79803$.

In the next section we shall adapt the Gamma three-parameter distribution to fit reduced volumes of Voronoi cells generated with Sobol sequence. The results will be compared with those obtained by a simpler PDF, a one parameter gamma

$$
p(x ; c)=\frac{c^{c}}{\Gamma(c)} x^{c-1} \exp (-c x),
$$

used by Kiang in his seminal work on Voronoi tessellations (Kiang, 1966), whose moments are

$$
\sigma^{2}=\frac{1}{c}, \quad \gamma=2 \frac{1}{\sqrt{c}}, \quad k=3 \frac{2+c}{c} .
$$

It has shown that good approximations for volume distributions of PVT cells can be obtained by setting $c=5$ (Ferenc \& Néda, 2007). A detailed comparison between $p(x ; c)$ and $G(x ; a, b, c)$ can be found in (Ferenc \& Néda, 2007), see also (Ferraro \& Zaninetti, 2012). 


\section{Volumes Statistics in SVT}

Sobol sequences, like all quasi-random sequences fill the space more uniformly than uncorrelated random points and this property has been extensively used in Monte Carlo methods such as integration or simulation of transport processes (Morokoff \& Caflisch, 1994). Indeed the uniformity of quasi-random sequences leads to integration errors smaller than in case of random sequences.

Evidence of uniformity of a Sobol sequence compared with a random one is presented in Figures 1 (155 random seeds) and 2 (140 quasi-random seeds) that show respectively a PVT and SVT. Here for the generation of points of the Sobol sequence we have used the procedure outlined in (Press, Teukolsky, Vetterling, and Flannery, 1992; Antonov \& Saleev, 1979), for clarity's sake the examples are two-dimensional and just 140 centers have been used. It is apparent from the Figures that SVT exhibits a narrower distribution of areas: indeed variances are 0.27 and 0.061 for PVT and SVT respectively.

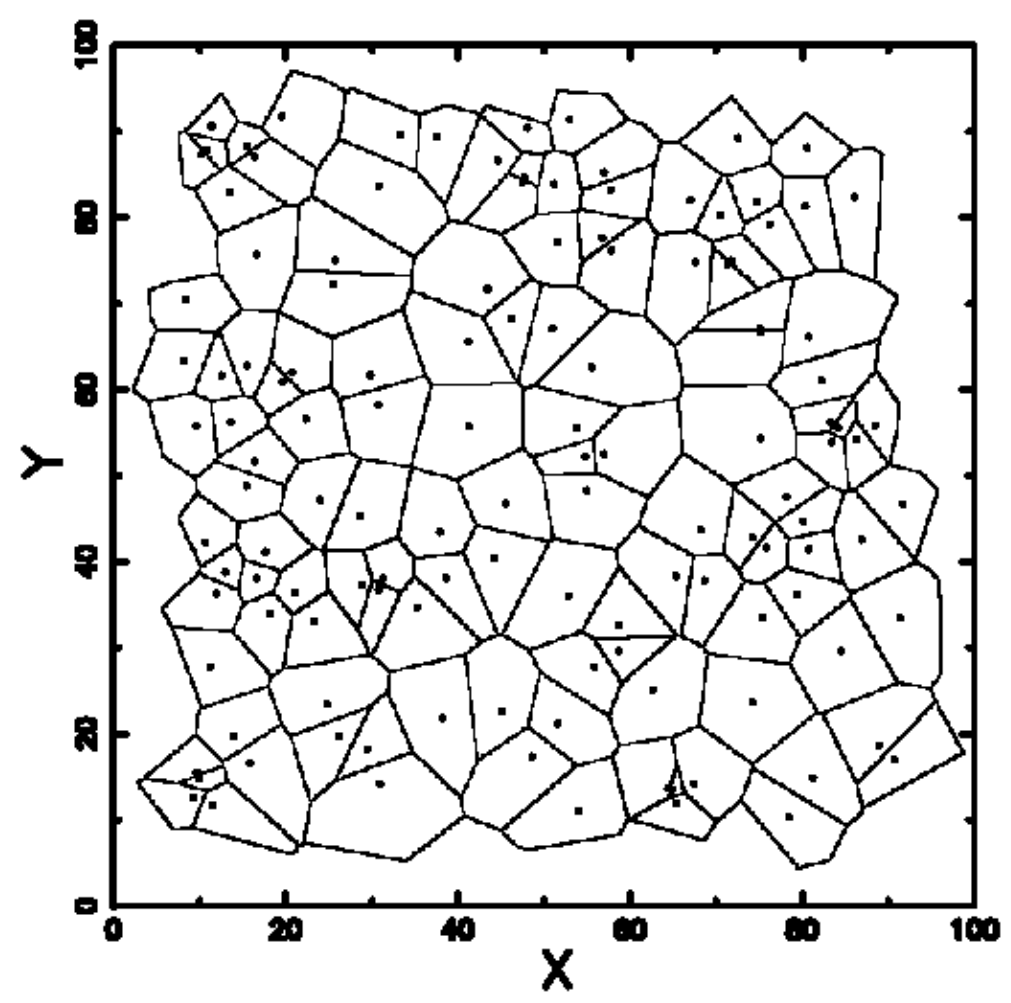

Figure 1. An example of PVT in 2D

A measure of uniformity of a quasi-random sequence is the discrepancy, which is the error made when representing the volume of subsets of the unit cube by the fraction of points in the subsets: the lower of the discrepancy the higher is the uniformity. There are different ways to define discrepancy see (Morokoff \& Caflisch, 1994) and references therein, and it can be shown that the discrepancy on a $d$ dimensional cube is roughly $(\log n)^{s} n^{-1}$ for a large number of points $n$, whereas random sequences have discrepancy of size $(\log \log n)^{1 / 2} n^{-1}$.

A simple measure of uniformity can be also defined as follows: for a Sobol tessellation with $n$ seeds of an unit area the minimum distance $d_{\min }$ between any two seeds is first computed and next $d_{\min }$ is divided by $d_{L}=\frac{1}{n}$, the distance between two seeds in the case of a regular lattice of unit area. Thus the ratio $\frac{d_{\text {min }}}{d_{L}}$ should be larger for a quasi-random sequence of seeds compared with a random one. When $n=1000$ the ratio $\frac{d_{\min }}{d_{L}}$ is 0.093 in the case of 2D Sobol seeds and 0.0056 in the case of random (Poissonian) seeds.

Thus, Sobol sequences present a repulsion effect that can be also found in other quasi-random sequences such as, for example, those generated by the eigenvalues of complex random matrices, see (Le Caer \& Ho, 1990). 


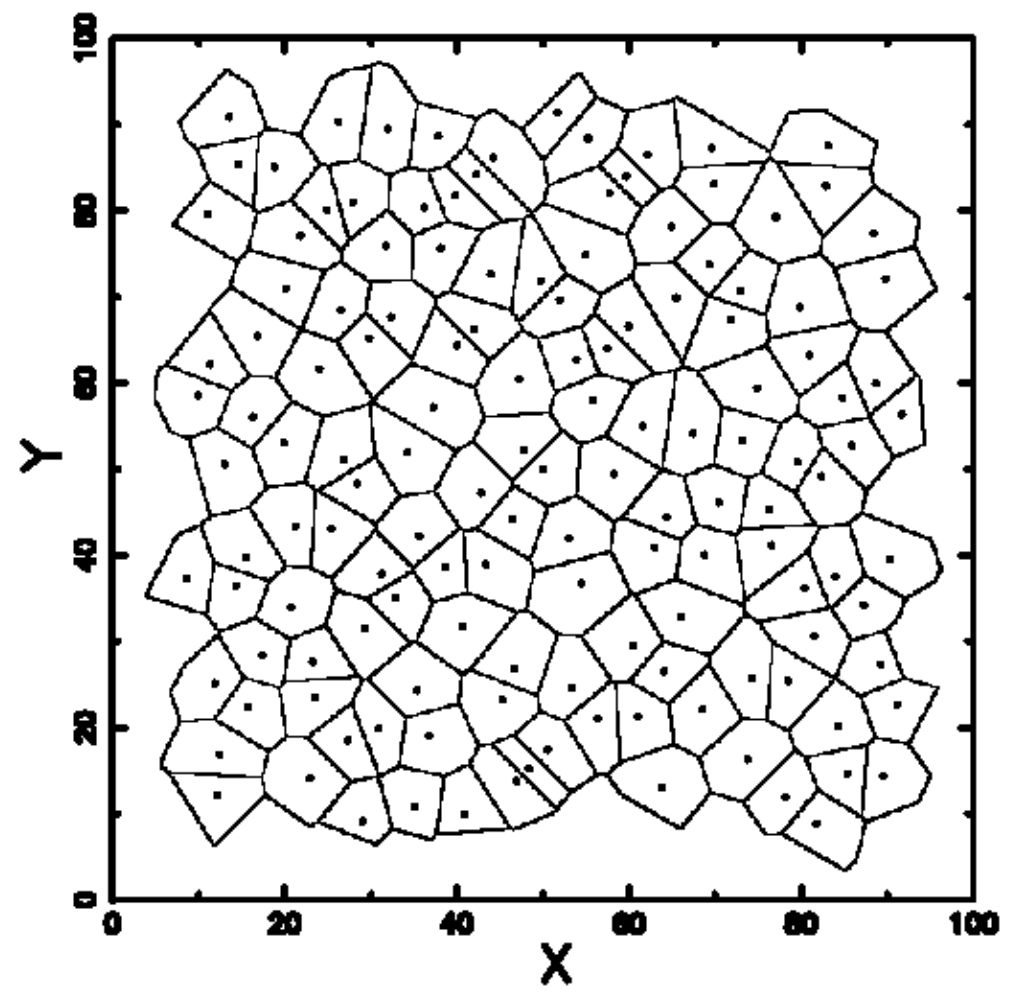

Figure 2. An example of SVT in 2D

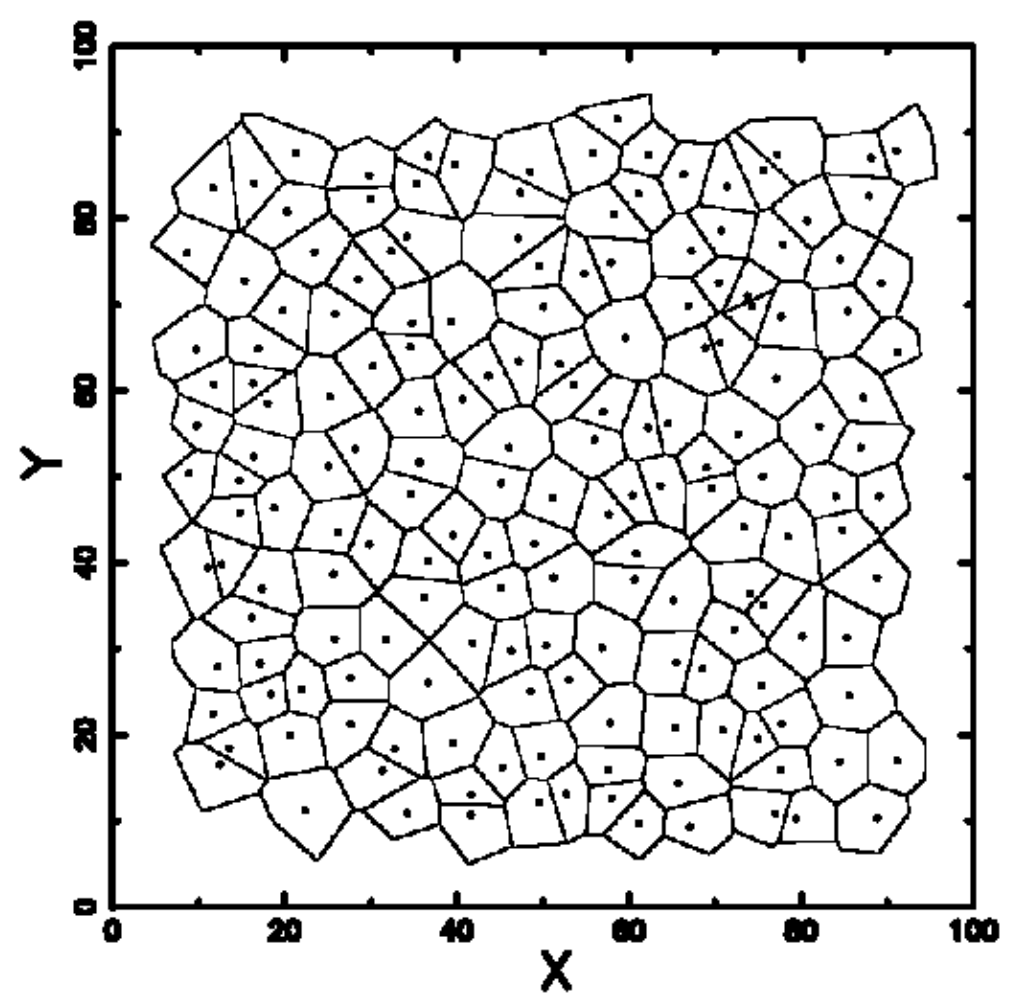

Figure 3. Tessellation generated by 174 eigenvalues from complex matrix in $2 \mathrm{D}$ 
The eigenvalues seeds can be found starting from a random $N \times N$ complex matrix. The matrix elements are given by $x+i y$ where $\mathrm{x}$ and $\mathrm{y}$ are pseudo random real numbers taken from a normal ( Gaussian ) distribution with mean zero and standard deviation $1 / \sqrt{2}$. Once obtained the complex elements we diagonalize the complex matrix using the subroutine CG from the EISPACK library. The points seeds have the $x$ and $y$ coordinates corresponding to the real and imaginary parts of the complex eigenvalues and an example which has variance 0.06 is reported in Figure 3.

Next we have generated a three-dimensional SVT with $10^{4}$ cells: the histogram of their volumes has been fitted with the generalized gamma $G(x ; a, b, c)$, as given by (2), and the one parameter gamma $p(x ; c)$, see (7), respectively.

The statistical parameters of the two fits and the sample's parameters are reported in Table 1, were the first line shows numerical values of the parameters for generalized three-parameter and the one-parameter gamma, respectively, obtained from the fit of empirical data, the next lines report values of mean, variance, skewness and the kurtosis for the two distributions and the sample, here the number of cells is $10^{4}$ and the number of bins is 40 .

Table 1. Parameters for generalized three-parameter and the one-parameter gamma, respectively, obtained from the fit of normalized volumes

\begin{tabular}{cccc}
\hline Moment & Generalized gamma & One parameter gamma & Sample \\
& $a=2.3317, b=2.86816, c=7.32528$ & $c=16.32099$ & \\
\hline$\left\langle x_{S V T}\right\rangle$ & 0.99993 & 1 & 1 \\
$\sigma_{S V T}^{2}$ & 0.0613809 & 0.06127 & 0.06127 \\
$\gamma_{S V T}$ & 0.191594 & 0.49505 & 0.20973 \\
$k_{S V T}$ & 2.94336 & 3.36762 & 3.15243 \\
\hline
\end{tabular}

The goodness of the fit has been assessed by first computing the PDF of both $G$ and $p$ and next applying the Kolmogorov-Smirnov (K-S) test (Kolmogoroff, 1941; Smirnov, 1948; Massey, 1951).

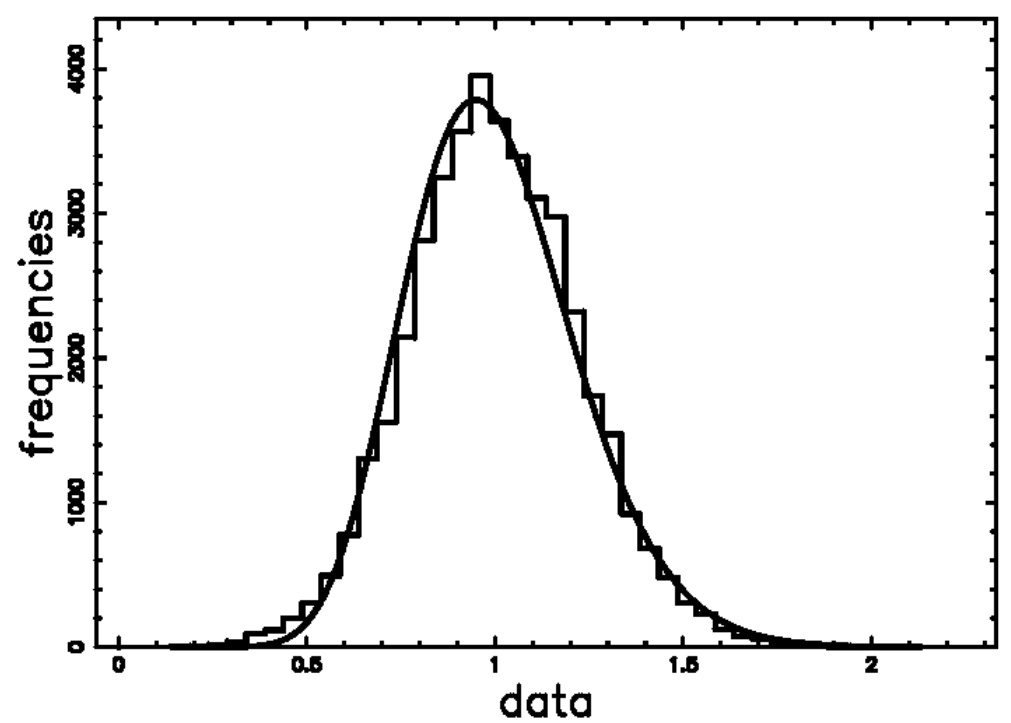

Figure 4. Histogram (step-diagram) of the SVT reduced volume distribution

The three- parameter PDF $G$ fits well the simulated volumes: the maximum distance between the empirical and computed distributions functions $d_{\max }=0.01$ and the result of the K-S test, implemented with the FORTRAN subroutine KSONE (Press, Teukolsky, Vetterling, and Flannery, 1992), gives $P_{K S}=0.21$. Note also that its moments appear to be close with those derived from the empirical distribution.

As concerns $\mathrm{p}(\mathrm{x}: \mathrm{c})$ the results of the K-S test are worse, $d_{\max }=0.024, P_{K S}=1.910^{-5}$. Figure 4 shows the histogram of volumes generated by the simulation, the number of Sobol centers is $10^{4}$, the number of division 
$\mathrm{n}=40$, and the graph of the generalized gamma used to fit the data with parameter values given by Table (1). In Figure 5 we report the comparison between the empirical distribution and the distribution function (DF) of $G$ with parameters as in Table 1. It can be interesting to compare values of the moments obtained here with those derived for PVT, by using estimate of $a, b, c$ given in (Tanemura, 2005), namely

$$
\sigma_{P V T}^{2}=0.1787603, \quad \gamma_{P V T}=0.7766972, \quad k_{P V T}=3.849375 .
$$

Volumes distribution of SVT shows a smaller variance, as result of the fact the centers are distributed in a more regular fashion than in case of PVT; furthermore the PDF is more symmetric for SVT $\left(\gamma_{S V T}<\gamma_{P V T}\right)$; finally $k_{S V T}$ is very close to 3 , the value of the Gaussian kurtosis.

A comparison between the generalized gamma PDFs for the two cases is shown in Figure 6.

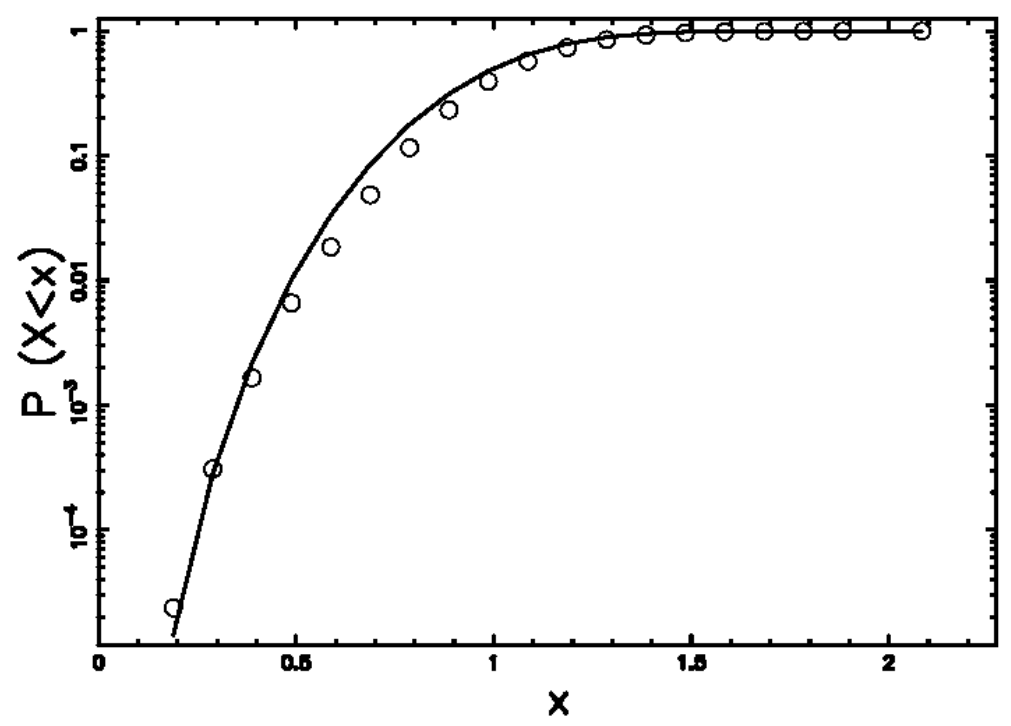

Figure 5. Simulated volume distribution (small circles) and DF of the generalized gamma (full line)

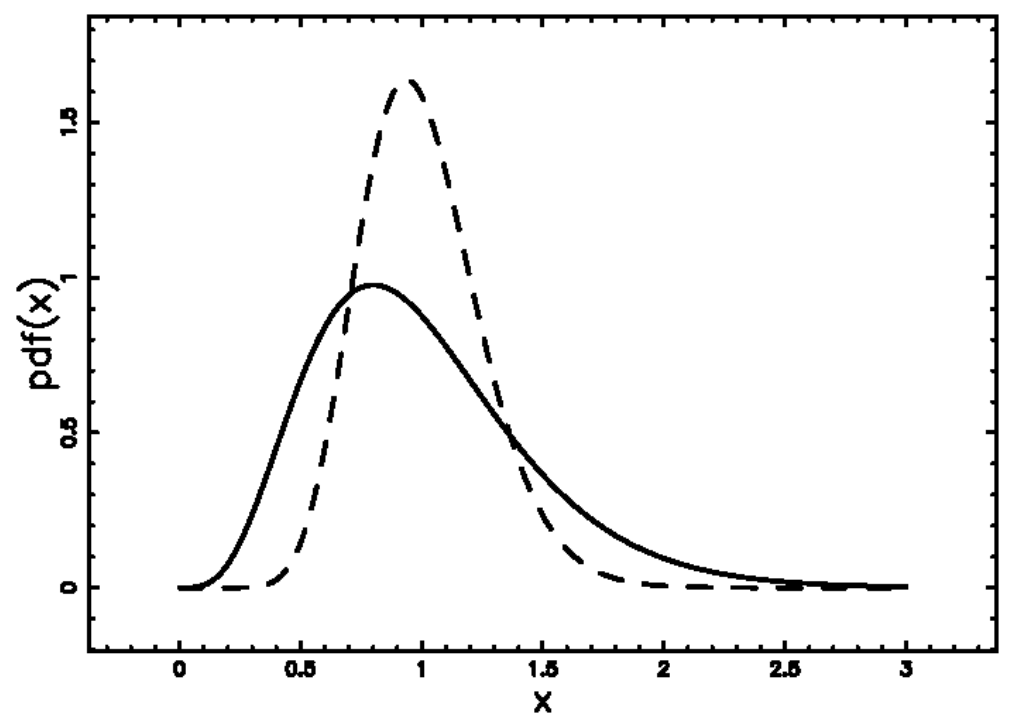

Figure 6. Plot of reduced gamma PDFs for the PVT (full line) and SVT case (broken line) 


\subsection{An application}

In this section it has been shown that the main differences between distributions of volumes in case of SVT vs PVT are that the former have a smaller variance and are more symmetric and it has been argued that, clearly, these differences are related to the more regular distributions of centers in case of SVTs. This suggests a possible application in understanding different PDF for volumes obtained in simulations of local structure of gases, in the liquid-gas phase. In (Idrissi, Vyalov, Damay, Kiselev, Puhovski, \& Jedlovszky, 2010) $\mathrm{CO}_{2}$ was considered and simulations were carried out to determine the volumes available to each molecule that were considered as the center of a Voronoi tessellation. From the simulation the empirical PDF of volumes $P(V)$ can be computed and results show an increase of mean volume $\langle V\rangle$ and standard deviation $\sigma_{V}$ as temperature rises: the former effect is due to the thermal expansion of the system (Idrissi, Vyalov, Damay, Kiselev, Puhovski, \& Jedlovszky, 2010), whereas the increase of $\sigma_{V}$ points to more disordered distribution of the centers at higher temperatures and to increasing volume fluctuations. To compare our results with the data in (Idrissi, Vyalov, Damay, Kiselev, Puhovski, \& Jedlovszky, 2010), see Table 2, we have computed the standard deviation for the reduced volumes $x=V /\langle V\rangle$,

Table 2. Mean values and standard deviations of the Voronoi polyhedra, $\mathrm{V}$, and $\mathrm{c}$ of the Kiang function

\begin{tabular}{cccccccc}
\hline $\mathrm{T} / \mathrm{K}$ & 250 & 270 & 285 & 298 & 303 & 306 & 313 \\
$\langle V\rangle / \AA^{3}$ & $69.7 \pm 10.3$ & $77.5 \pm 13.8$ & $87.1 \pm 19.4$ & $105.3 \pm 31.1$ & $114.4 \pm 38.2$ & $156.8 \pm 68.8$ & $156.8 \pm 65.1$ \\
$\mathrm{c}$ & 45.79 & 31.53 & 20.15 & 11.46 & 8.96 & 5.19 & 5.8 \\
\hline
\end{tabular}

namely $\sigma=\sigma_{V} /\langle V\rangle$, and compared them with the standard deviations $\sigma_{S V T}$ and $\sigma_{P V T}$ of the PDFs derived in. As an example when $T=250 K,\langle V\rangle=69.7 \AA^{3}, \sigma=10.3 / 69.7=0.147, \sigma^{2}=0.0218$ and $c=1 / 0.0218=45.79$. We briefly recall that the data of the previous Table are theoretical values computed with the Voronoi polyhedra (VP) analysis based on numerical codes developed by (Jedlovszky, 1999, 2000; Tokita, Hirabayashi, Azuma, \& Dotera, 2004).

In the temperature range from $T=250$ to $T=303 \sigma$ increases from 0.15 to 0.33 and $\sigma_{S V T}=0.25$ is in the middle of this range. At higher temperatures, $T=303$ and $T=313$ one obtains $\sigma=0.43$ and $\sigma=0.41$, respectively, matching closely the standard deviation derived for for PVT, namely $\sigma_{P V T}=0.43$. More importantly, the shape of the $P D F$, which is quite symmetric at the lower end of the $T$ range, increasingly deviates symmetry as $T$ increases and develops an exponentially decaying tail at high volume values (Idrissi, Vyalov, Damay, Kiselev, Puhovski, \& Jedlovszky, 2010). This is also what happens in the transition from SVT to PVT, see Figure 6. These results can be explained as follows: as $T$ increases positions of molecules become more random and a transition takes place from a PDF relatively narrow and symmetric (like in the SVT case) to a more asymmetric PDF with larger variance corresponding to a PVT. The relation between the distributions of occupied volumes and the temperature $T$ can be made clearer by considering the parameter $c$ of the Kiang distribution (7). It is clear that small values of $c$ characterize distributions with relatively large variance and skweness, whereas as $c$ increase distributions become narrower and more simmetric. The parameter $c$ of the Kiang function can be parameterised as function of the temperature as follows

$$
c=C_{1} T^{\alpha_{1}},
$$

where $C_{1}$ and $\alpha_{1}$ can be found from the data of Table 2. A numerical procedure gives $C_{1}=5.710^{25}$ and $\alpha_{1}=-10$.

\section{Chords Length Distribution}

In many experimental conditions it is not possible to directly observe the three-dimensional cells forming a tessellation, just their linear sections: thus it is of interest to study the relationships between the geometric properties of three-dimensional structures and their chords (Ruan, Litt, \& Krieger, 1988; Okabe, Boots, Sugihara, and Chiu, 2000; Stoyan, Wagner, Hermann, \& Elsner, 2011).

One can distinguish three main ways to generate chords (Coleman, 1969), (Kellerer, 1984): isotropic uniform randomness results when the body is exposed to an uniform isotropic flow of infinite straight lines; weighted randomness occurs when a uniformly distributed random point is chosen and is traversed by a straight line with uniform random direction; two-point randomness is obtained when a straight line traverses two random points that are independently and uniformly distributed. The first case, which will be considered here since more relevant for practical applications (Kellerer, 1984); relations among PDFs of chords generated by different methods can be found in (Kellerer, 1984). 
In order to obtain formulas for the distributions of the chords generated by the intersections of lines with SVTs some simplifications are needed: here the polyhedrons forming the cells will be approximated by spheres and the one-parameter distribution $p$ as given by Equation(7), will be used to fit the cells volumes distribution. With these assumptions a formula for the PDF of chords length can been obtained, a simple iterative procedure will then be used to adapt this distribution to the simulated data, thus correcting the errors resulting from the approximations. Let $p_{x}$ be the probability density function for the reduced cell volumes, then the PDF $p_{y}$ for the lengths of the diameters is given by

$$
p_{y}(y)=\frac{\pi}{2} p_{x}\left(\frac{1}{6} \pi y^{3}\right) y^{2},
$$

and the probability density function $g$ of chords length $l$ is

$$
g(l)=\frac{2 l}{\left\langle y^{2}\right\rangle} \int_{l}^{\infty} p_{y}(y) d y
$$

see, for instance, (Ruan, Litt, \& Krieger, 1988), (Watson, 1971). In the present case then, with PDF of volumes given by the Kiang function, Equation (7), with $c=16$, the distribution of diameters is

$$
p_{y}(y)=\frac{1}{2} \frac{16^{16} \pi^{16}}{6^{15} \Gamma(16)} y^{47} \exp \left(-\frac{8 \pi}{3} y^{3}\right)
$$

The use of the generalized gamma function, Equation(2), for the PDF in volumes means conversely that the integrals which follow can be done only in a numerical way. The probability density $g_{S V T}$ can be found by making use of Equation (11) with $p_{y}$ given by (12), the result is

$$
g_{S V T}(l)=\frac{a_{0}}{a_{1}} \exp \left(-\frac{8}{3} \pi l^{3}\right) \sum_{k=0}^{9} b_{k} \pi^{k} l^{3 k+1},
$$

where the coefficients are large numbers, whose values are reported in the Appendix. The previous formula and the following ones are not an exact analytical result but results from the approximation of the volume of the Voronoi's polyhedrons by spheres. In order to check the validity of this PDF we inserted in a box 50000 seeds which produce a network of irregular faces belonging to the Voronoi's polyhedra. We selected 120 random lines which will intercept the network of the irregular faces: the chord's length is evaluated as the distance between a face and the following one on the considered line. A typical run processes a total number of $\approx 3200$ chords. The corresponding histogram is shown in Figure 7: note that here the results has been rescaled so that the average value of chord length is equal to 1 .

It is apparent that the results of the simulations do not agree with the PDF given by Equation (13): it is enough to note that $g_{S V T}(0)=0$, is in contrast with the histogram of Figure 7. In order to overcome this problem a new variable $z$ has been defined by a shift of $l: z=l-a$, so that $g_{1, S V T}(z)=g_{S V T}(z+a)$. The shift parameter $a$ should not be confused with the parameter of the generalized gamma PDF (compare Equation (2)). An explanation for this shift is given by the fact that the length of the chord which touches only in one point the sphere is zero conversely a chord which lies on a irregular face of the Voronoi's polyhedron has a finite length. This means that we have more short lengths in the simulation of the chords with the real polyhedrons in respect to the length's of theoretical intersections with the spheres: i.e. the PDF of having short chords is finite rather than zero.

Next, to obtain a reduced variable, a scale change has been applied resulting in $u=b z$. The scale parameter $b$ used here is different from the parameter of the generalized gamma PDF (compare Equation (2)). 
Table 3. Moments of the probability density function $g_{f, S V T}$, Sobol seeds

\begin{tabular}{ll}
\hline Parameter & value \\
\hline Mean & 1 \\
Variance & 0.308 \\
Skewness & 0.0888 \\
Kurtosis & 2.164 \\
\hline
\end{tabular}

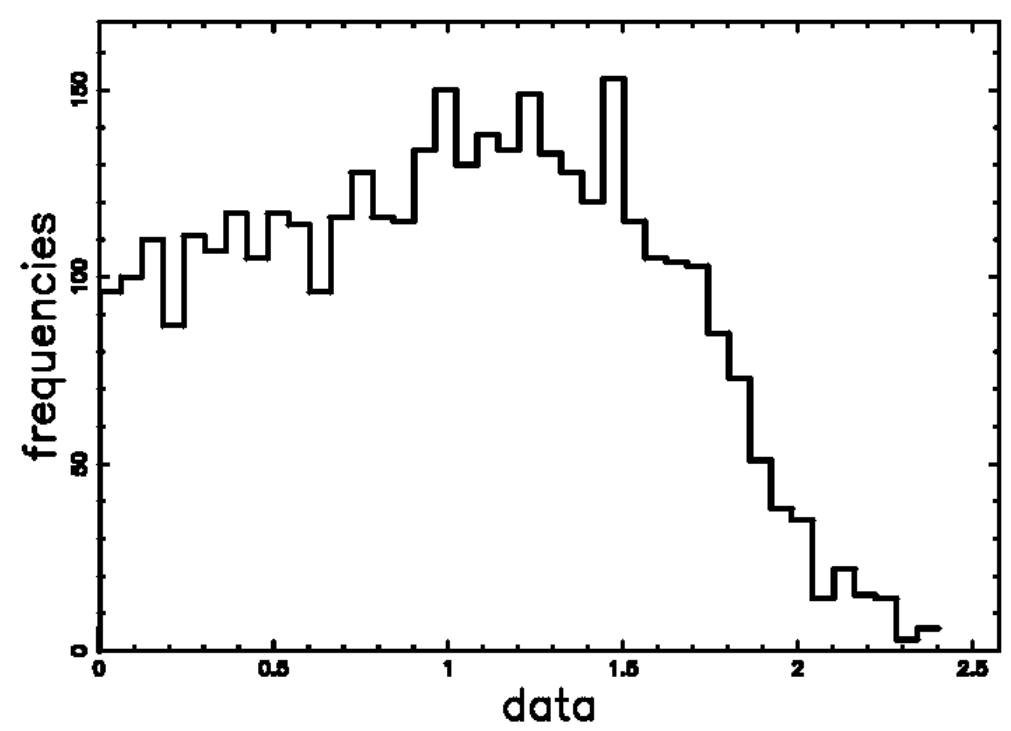

Figure 7. Histogram (step-diagram) for SVT chord length with average value 1

In conclusion, following translation and scale change, the final PDF is now

$$
\begin{aligned}
g_{f, S V T}(u ; a, b) & =\frac{C}{b} g_{S V T}\left(\frac{u}{b}+a\right) \\
& =\frac{C a_{0}}{b a_{1}} \exp \left(-\frac{8}{3} \pi\left(\frac{u}{b}+a\right)^{3}\right) \sum_{k=0}^{9} b_{k} \pi^{k}\left(\frac{u}{b}+a\right)^{3 k+1},
\end{aligned}
$$

where $C$ is a normalizing constant, which has value 1.4717 .

Numerical values of $a$ and $b$ have been obtained by an interactive procedure that at each step computes the DF

$$
F(u ; a, b)=\int_{0}^{u} g_{f, S V T}(z ; a, b) d z \quad:
$$

the agreement between the calculated and simulated distribution function is then been verified by the K-S test. The procedure is halted when $d_{\max }$, the maximum distance between the distribution functions, reaches a minimum (that is when the significance level $P_{K S}$ is maximum) and the corresponding pair $a, b$ is selected; Table 4 reports the adopted values. Calculated and empirical DFs of chords length are shown in Figure 8, the K-S test gives $d_{\max }=$ $0.0165, P_{K S}=0.251$. The moments of $g_{f, S V T}$ are presented in Table 3 with parameters as in Table 4 .

The results can also be presented as a PDF, see Figure 9, parameters as in Table 4; the reduced $\chi^{2}$ is 1.09. 


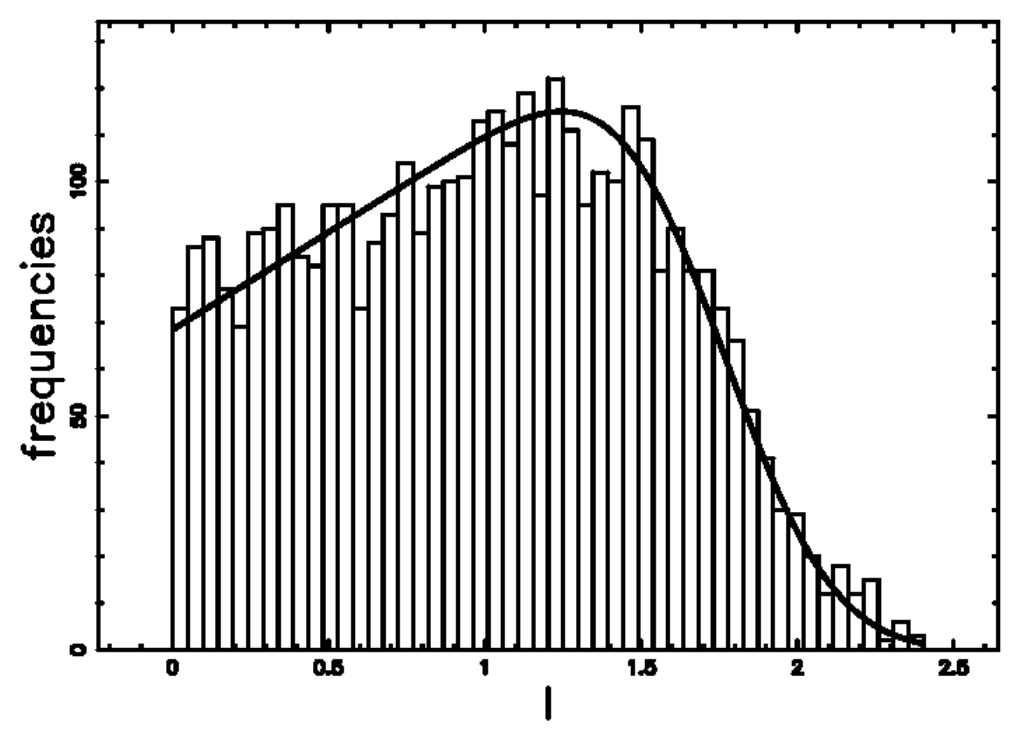

Figure 9. Histogram (step-diagram) for SVT chord length with average value 1 and PDF $g_{f, S V T}$ ( line)

The shifted PDF, $g_{f, S V T}$, for SVT chords can be reported as a Taylor expansion around $\mathrm{x}=0$, when the average value is one

$$
\begin{array}{r}
g_{f, S V T}(x ; 0.7,3.27)=0.411+0.179 x-0.44410^{-5} x^{2} \\
-0.24310^{-4} x^{3}-0.95810^{-3} x^{4}-0.28510^{-3} x^{5} .
\end{array}
$$

It is clear that at $x=0$ this PDF takes a finite value.

\subsection{The PVT Chord}

Chords length distribution in case of PVT can be obtained with the same method, by adopting now as the volumes PDF Equation (7) with $c=5$, which is known to give a good fit of simulated data (Ferenc \& Néda, 2007). The resulting integral for the chord as given by eqn.(11) is

$$
\begin{array}{r}
g_{P V T}(l)=\frac{125 l^{13} \pi^{14 / 3} 5^{2 / 3} \sqrt[3]{6}}{39424 \Gamma(2 / 3)\left(\mathrm{e}^{\pi l^{3}}\right)^{5 / 6}}+\frac{75 l^{10} \pi^{11 / 3} 5^{2 / 3} \sqrt[3]{6}}{4928 \Gamma(2 / 3)\left(\mathrm{e}^{\pi l^{3}}\right)^{5 / 6}} \\
+\frac{135 l^{7} \pi^{8 / 3} 5^{2 / 3} \sqrt[3]{6}}{2464 \Gamma(2 / 3)\left(\mathrm{e}^{\pi l^{3}}\right)^{5 / 6}}+\frac{81 l^{4} \pi^{5 / 3} 5^{2 / 3} \sqrt[3]{6}}{616 \Gamma(2 / 3)\left(\mathrm{e}^{\pi l^{3}}\right)^{5 / 6}} \\
+\frac{243 l \pi^{2 / 3} 5^{2 / 3} \sqrt[3]{6}}{1540 \Gamma(2 / 3)\left(\mathrm{e}^{\pi / 3}\right)^{5 / 6}} .
\end{array}
$$

We now apply translation and scale change

$$
g_{f, P V T}(u ; a, b)=\frac{C}{b} g_{P V T}\left(\frac{u}{b}+a\right),
$$

and Table4 reports the parameters adopted. Figure 10 reports a comparison of the previous result, integration of PDF (18)and parameters as in Table 4, as a dashed line with the tabulated result as deduced from Table 5.7.4 in (Okabe, Boots, Sugihara, and Chiu, 2000) when the average value of both PDFs is one. Table 5 reports the two numerical sequences.

Calculated and empirical DFs of chords length in the PVT case are shown in Figure 11 with parameters as in Table 4; the K-S test gives $d_{\max }=0.0362$.

For comparison purposes a plot of $\operatorname{PDF} g_{f}(u ; a, b)$, which represents the SVT case with parameters as in Table 4, is shown in Figure 12 together with the numerical PDF for PVT derived from the numerical DF reported in Table 5.7.4 of (Okabe, Boots, Sugihara, and Chiu, 2000); in both cases the mean chord length is equal to 1. 


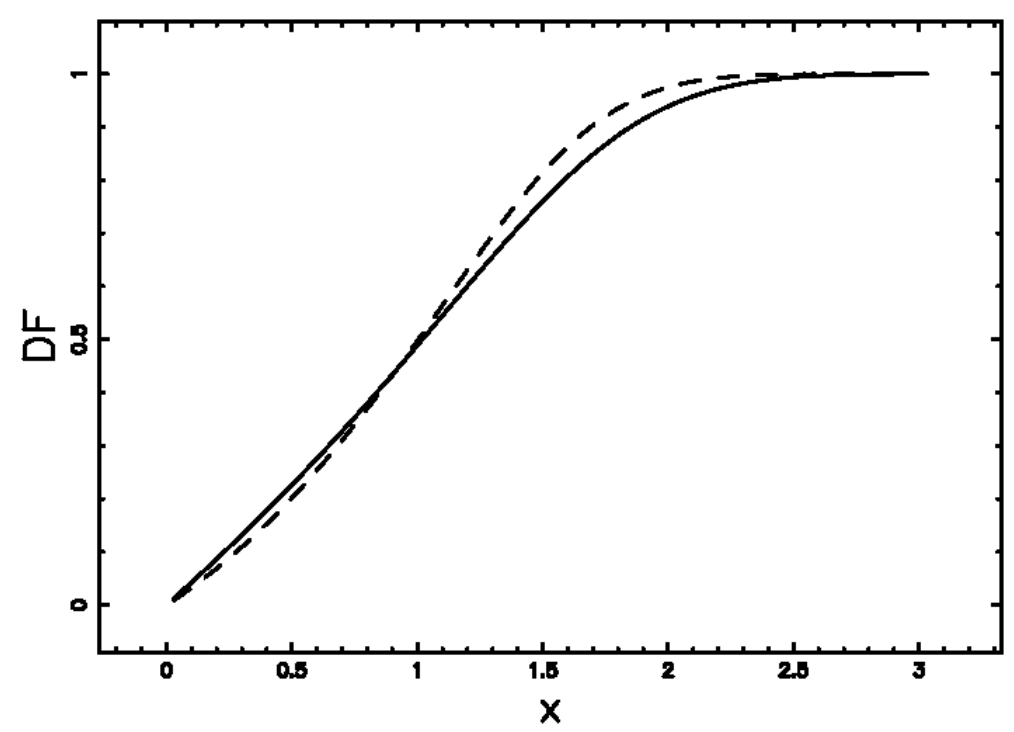

Figure 10. The full line represents the tabulated chord DF in the case of PVT, the dashed line is our chord DF

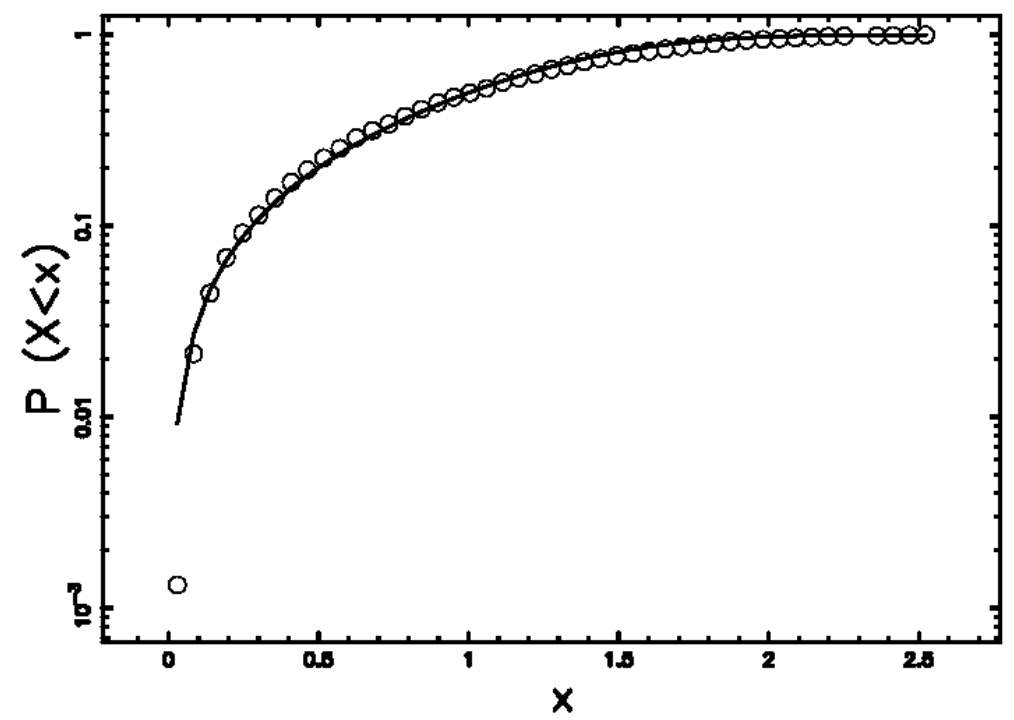

Figure 11. Comparison between data (empty circles) and theoretical DF for $g_{f, P V T}$ (continuous line) of chords length distribution 
Table 4. Parameters which characterize the chord distribution according to the chosen seed

\begin{tabular}{cccc}
\hline type of seed & $\mathrm{a}$ & $\mathrm{b}$ & $\mathrm{C}$ \\
\hline Poissonian seeds & 0.4 & 1.944413550 & 0.8937531655 \\
Sobol seeds & 0.7 & 3.271926163 & 1.471732407 \\
ACG seeds,s $=0.64$ & 0.7 & 3.271926163 & 1.471732407 \\
\hline
\end{tabular}

Table 5. Tabulated chord length DF for PVT case (second column) and our DF (third column)

\begin{tabular}{ccc}
\hline$x$ & PVT DF & our DF \\
\hline 0.3 & 0.1320 & 0.1091 \\
0.6 & 0.2760 & 0.2518 \\
0.9 & 0.4336 & 0.4298 \\
1.2 & 0.6008 & 0.6306 \\
1.5 & 0.7602 & 0.8139 \\
1.8 & 0.8844 & 0.9331 \\
2.1 & 0.9579 & 0.9851 \\
2.4 & 0.9891 & 0.9986 \\
2.7 & 0.9981 & 0.9985 \\
3.0 & 0.9998 & 0.9983 \\
\hline
\end{tabular}

The shifted PDF, $g_{f, P V T}$, for PVT chords is reported as a Taylor expansion around $x=0$, when the average value is one

$$
\begin{array}{r}
g_{f, P V T}(x ; 0.563,2.452)=0.386+0.279 x-0.00257 x^{2} \\
-0.00766 x^{3}-0.0151 x^{4}-0.02 x^{5} .
\end{array}
$$

\section{Adjustable Seeds}

An occasional reader may question if a scenario of gradual transition from PVT to SVT can be outlined. In order to have more flexible seeds we introduce the adjustable Cartesian grid (ACG) which can be computed both in 2D and 3D.

The algorithm is now outlined:

1). The process starts inserting the seeds on a 2D/3D regular Cartesian grid with equal distance $\delta$ between one point and the following one

2). A random radius is generated according to the half Gaussian , $H N(x)$, which is defined in the interval $[0, \infty]$

$$
H N(x ; s)=\frac{2}{s(2 \pi)^{1 / 2}} \exp \left(-\frac{x^{2}}{2 s^{2}}\right) \quad 0<x<\infty .
$$

The main moments of $H N(x ; s)$ are:

$$
\langle x\rangle=\frac{s \sqrt{2}}{\sqrt{\pi}},
$$

and

$$
\sigma^{2}=\frac{s^{2}(\pi-2)}{\pi}
$$

3). A random direction is chosen in $2 \mathrm{D} / 3 \mathrm{D}$ and the two/three Cartesian coordinates of the generated radius are evaluated. These two/three small Cartesian components are added to the regular 2D/3D grid which represent the seeds. In order to have small corrections we express $s$ in $\delta$ units. The parameter $s$ is a good "disorder 


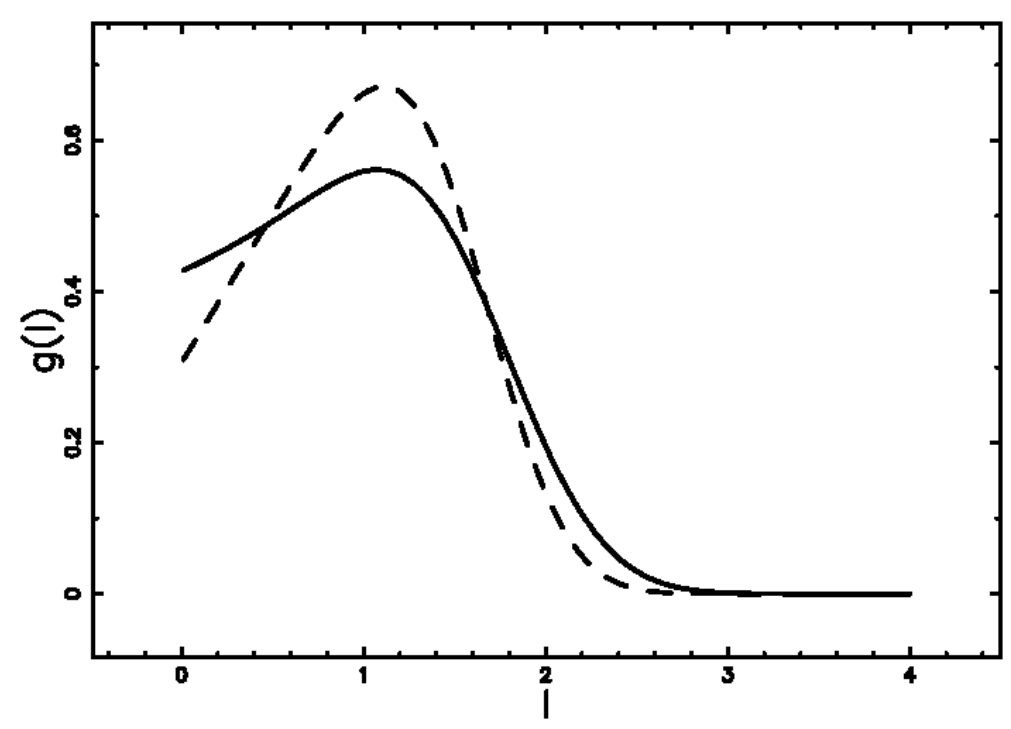

Figure 12. The full line represents the PDF of chords length in case of PVT, the dashed line is the graph of $g_{f, S V T}$

parameter" for the generated configurations. At $s=0$ we will have the seeds disposed on a perfect lattice with all the volumes of the irregular polyhedra equal, increasing $\mathrm{s}$ we will reach before $\mathrm{c}=16$ in the PDF of volumes (SVD) and subsequently c=5 (PVT).

Figure 13 reports an example of 2D tessellation from ACG which areas have variance 0.047 , the same value of the $2 \mathrm{D}$ sobol seeds.

\subsection{Applications of the Adjustable Seeds}

We present here two applications of the adjustable seeds. These adjustable $3 D$ seeds can be calibrated in order to have $c=16$ for the Kiang distribution in volumes, see PDF (7). To each value of $s$ corresponds one value of $c$ that can be obtained from the relation $c=1 / \sigma^{2}$. For instance when $s=0.316, c=32.82$ and at when $s=1, c=15.87$; in particular $c=16$ is obtained for $s=0.64$. The first application refers again to the local structure of gas $\left(\mathrm{CO}_{2}\right)$ in the liquid-gas coexistence phase (Idrissi, Vyalov, Damay, Kiselev, Puhovski, \& Jedlovszky, 2010). The parameter $c$ of the Kiang function for the PDF in volumes can be parameterised as a function of the parameter $s$ as follows

$$
c=C_{2} s^{\alpha_{2}},
$$

where $C_{2}$ and $\alpha_{2}$ can be found from a simulation. A numerical procedure gives $C_{2}=24.39$ and $\alpha_{2}=-0.44$. On equalizing the two equations (9) and (23) we obtain the following relationships between temperature, $T$, and regulating parameter $s$

$$
\begin{aligned}
& T=\left(\frac{C_{2}}{C_{1}}\right)^{\frac{1}{\alpha_{1}}} S^{\frac{\alpha_{2}}{\alpha_{1}}}, \\
& s=\left(\frac{C_{1}}{C_{2}}\right)^{\frac{1}{\alpha_{2}}} T^{\frac{\alpha_{1}}{\alpha_{2}}} .
\end{aligned}
$$

The previous relationship allows to find the theoretical standard deviation of the Voronoi polyhedra volumes as function of the temperature. We first fix the temperature as given by the values in Table 2 and the relationship (25) allows to find $s$. Given $s$ we obtain $c$ from eqn.(23) and by the fact that for the Kiang's function $\sigma^{2}=\frac{1}{c}$ we easily obtain $\sigma$ for a for the normalized variable. The standard deviation for the non-normalized variable is $\sigma_{V}=\langle V\rangle \sigma$. Table 6 reports the mean values and standard deviations of the Voronoi polyhedra, $V$, as computed in (Idrissi, Vyalov, Damay, Kiselev, Puhovski, \& Jedlovszky, 2010) (first line) and the procedure for the calculation of standard deviations presented here (second line). In both lines $V$ are the values as given in (Idrissi, Vyalov, Damay, Kiselev, Puhovski, \& Jedlovszky, 2010) and are presented here to make the comparison easier. The third and fourth line present the values of $c$ of the Kiang function and the regulating parameter $s$ of the ACG seeds. Figure 14 reports the chemical standard deviation as given by Chemistry and the theoretical standard deviation 


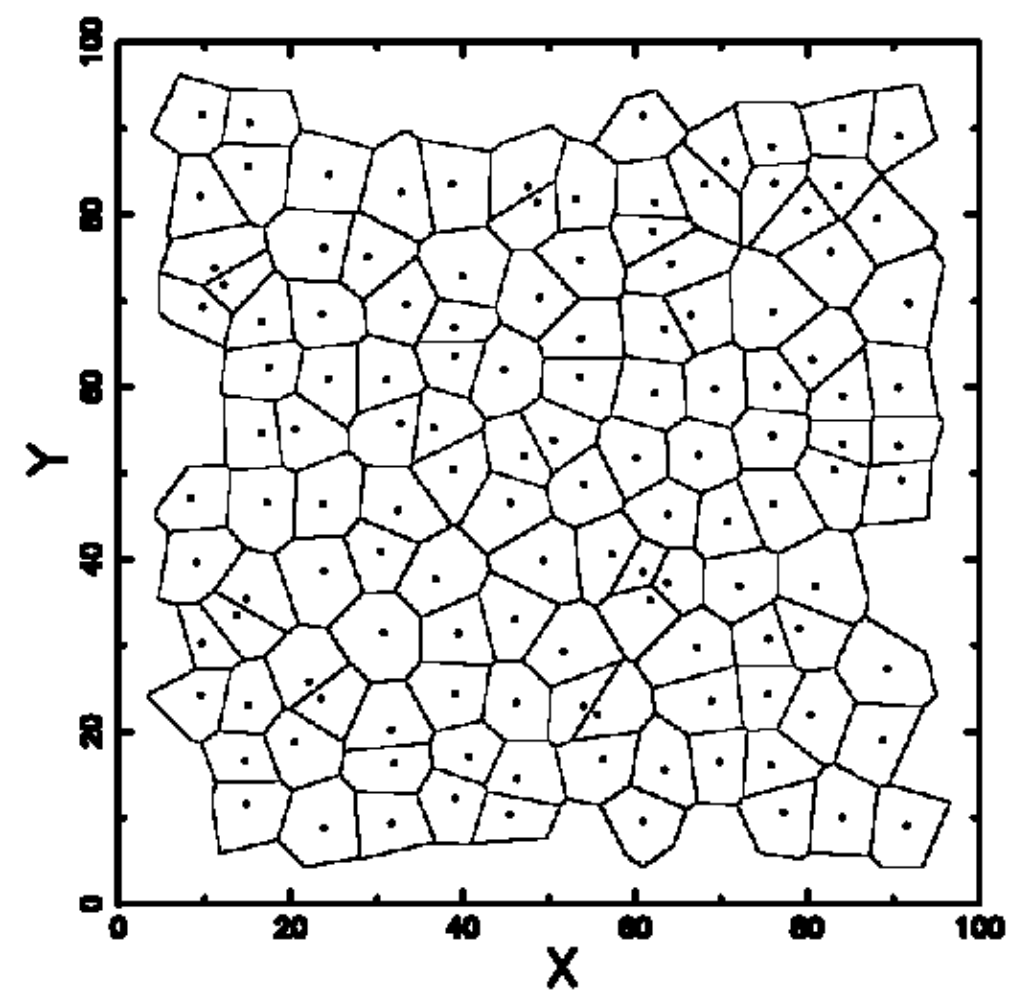

Figure 13. An example of 2D tessellation generated by 127 ACG seeds

as given by ACG as function of the temperature; it is clear that there is a good agreement between the calculated $\sigma_{V}$ and the experimental standard deviation. Next we consider the chords distribution of ACG tesselations, and

Table 6. Mean values (first line), standard deviations (second line), $c$ of the Kiang function (third line) and the regulating parameter $s$ of the ACG seeds (fourth line)

\begin{tabular}{cccccccc}
\hline $\mathrm{T} / \mathrm{K}$ & 250 & 270 & 285 & 298 & 303 & 306 & 313 \\
\hline$\langle V\rangle / \AA^{3}$ Idrissi et al. & $69.7 \pm 10.3$ & $77.5 \pm 13.8$ & $87.1 \pm 19.4$ & $105.3 \pm 31.1$ & $114.4 \pm 38.2$ & $156.8 \pm 68.8$ & $156.8 \pm 65.1$ \\
$\langle V\rangle / \AA^{3}$ & $69.7 \pm 9.19$ & $77.5 \pm 15.032$ & $87.1 \pm 22.142$ & $105.3 \pm 33.463$ & $114.4 \pm 39.51$ & $156.8 \pm 56.89$ & $156.8 \pm 63.7$ \\
$\mathrm{c}$ & 57.4 & 26.5 & 15.4 & 9.9 & 8.38 & 7.5 & 6 \\
$\mathrm{~s}$ & 0.143 & 0.823 & 2.808 & 7.729 & 11.275 & 14.101 & 23.56 \\
\hline
\end{tabular}

to this end we adapt the PDF of Sobol's chords as given by (15). Calculated and empirical distribution functions of chords length for ACG with parameters as in Table 4 are shown in Figure 15; the K-S test gives $d_{\max }=0.028$, $P_{K S}=0.0051$.

\section{Conclusion}

In this paper new types of three-dimensional Voronoi tessellations have been presented whose centers are not sampled from an uniform distribution, as in Poisson Voronoi (PVT) case, but rather are derived from more regular sequences. First Sobol-Voronoi Tesselations (SVT) have been considered in which cells forming the partition have as centers points generated by a Sobol quasi-random sequence. To make the notion of regularity of seeds configurations more precise a measure of uniformity has been presented. In analogy with the case of PVT we have used a generalized gamma distribution, denoted with $G_{S V T}$, to fit the volumes obtained by numerical simulations. One should expect that the more regular configuration of centers in the SVT is reflected in the distributions of cell volumes and this is indeed the case: comparisons between the PDFs show that $G_{S V T}$ have smaller variance and are more symmetric than the distribution $G_{P V T}$ of volumes in respect to PVT. It should be noted that volumes distributions of PVT cells can be fitted satisfactorily by different types of gamma distributions, as mentioned in Section 2; in contrast for volumes of SVT only the generalized three-parameters gamma provides a good fit. 


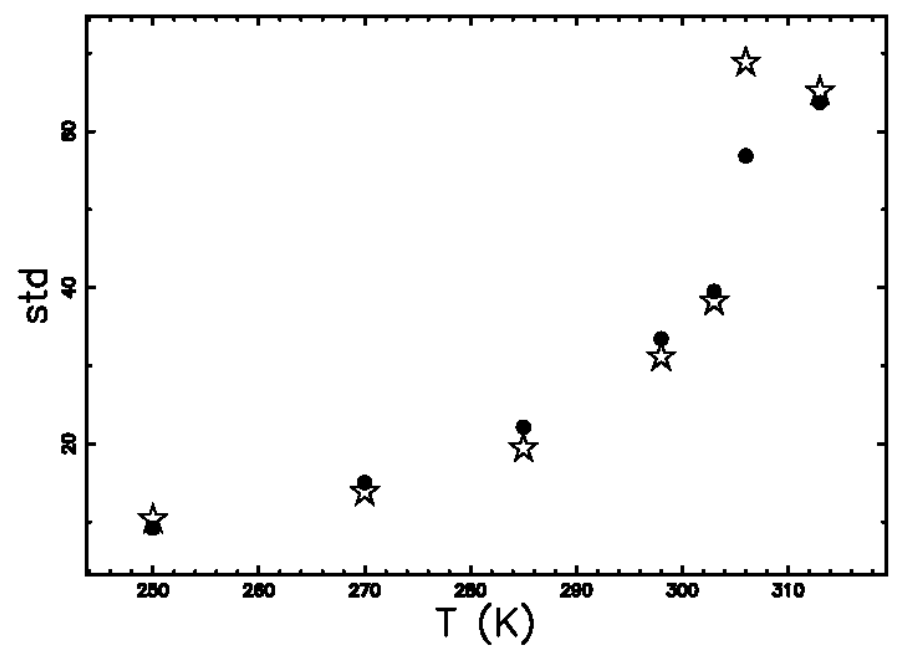

Figure 14. Standard deviation as given by Chemistry (empty stars) and theoretical standard deviation as given by variables volumes in ACG (full points) as function of the temperature.

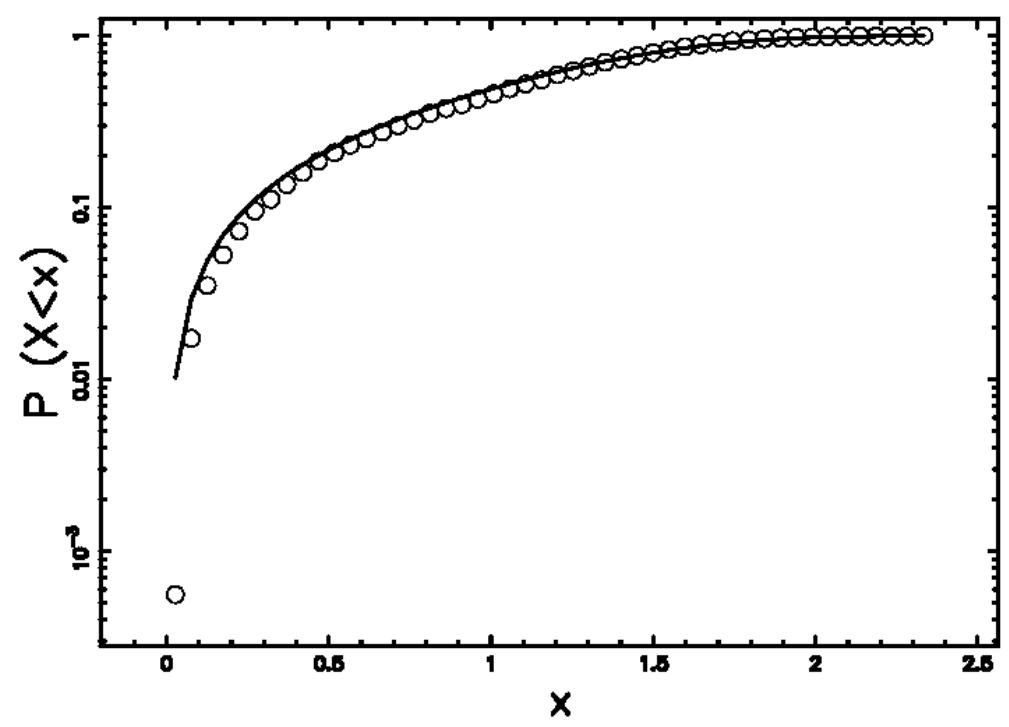

Figure 15. Data (empty circles) and theoretical DF for ACG seeds. The theoretical DF is the integral of PDF $\left(g_{f, S V T}\right)$ (continuous line). 
As concerns applications, SVT may be relevant in modelling partitions of systems that follow a more regular distribution than the usual Poisson distribution and, what is more interesting, transitions from ordered to disordered states of the system must be mirrored by a corresponding change from SVT to PVT. An example has been presented in which volumes occupied by molecules of $\mathrm{CO}_{2}$ in liquid-gas phases undergo a transformation from regular to more random distributions as the temperature increases. Transitions from regular to disordered partitions of space can be cast in a more general setting by considering the case in which center are first situated on the nodes of a regular grid and then positions are perturbed with a gaussian noise regulated by a parameter disorder $s$, thus creating an Adjustable Cartesian Grid (ACG). By increasing $s$ one can reach first SVT distributions and next PVT. In this case a transition from ordered to disordered states can be parametrized by $s$. Considering again to local structure of $\mathrm{CO}_{2}$ a relation has been derived between $s$ and $T$, from which the standard deviation has been computed and next compared with the experimental one, showing a good agreement. Finally statistics of chords resulting from intersections of line with elements of PVT, SVT and ACG have also been investigated. The interest of such type of statistics resides in the fact that in many experimental conditions only chords of three-dimensional cells can be determined. Results show a good agreement between the analytical formula, obtained with a semiempirical procedure and data obtained from a simulation, despite the approximations that have been used, namely considering cells to be a sphere and using an one-parameter gamma distribution to fit cells volumes, from which chords distributions have been derived.

\section{Appendix}

Numerical values of coefficients in Equation (13).

$$
\begin{gathered}
a_{0}=\pi^{2 / 3} 8^{2 / 3} \sqrt[3]{3}, \quad a_{1}=63997774118278000 \Gamma(2 / 3) . \\
b_{0}=9161961861677625, \quad b_{1}=24431898297807000, \quad b_{2}=32575864397076000, \\
b_{3}=28956323908512000, \quad b_{4}=19304215939008000, \quad b_{5}=10295581834137600, \\
b_{6}=4575814148505600, \quad b_{7}=1743167294668800, \quad b_{8}=581055764889600, \\
b_{9}=172164671078400, \quad b_{10}=45910578954240, \quad b_{11}=11129837322240, \\
b_{12}=2473297182720, \quad b_{13}=507343011840, \quad b_{14}=96636764160 \\
b_{15}=17179869184 .
\end{gathered}
$$

$\mathrm{k}$

\section{References}

Antonov, I., \& Saleev, V. (1979). An economic method of computing LP-sequences. U.S.S.R. Comput. Math. Math. Phys., 19, 252-256.

Blower, J., Keating, J., Mader, H., \& Phillips, J. (2002). The evolution of bubble size distributions in volcanic eruptions. Journal of Volcanology and Geothermal Research, 120, 1 - 23.

Bratley, P., \& Fox, B. L. (1988). Implementing Sobol's quasirandom sequence generator. ACM Trans. Math. Softw., $14,88-100$.

Chiu, S. N., \& Quine, M. P. (2001). Central limit theorem for germination-growth models in $\mathrm{R}^{* *} \mathrm{~d}$ with non-Poisson locations. Adv. Appl. Probab., 33, 751-755.

Coleman, R. (1969). Random Paths through Convex Bodies. Journal of Applied Probability, 6, 430-441. 
Du, Q. and Wang, D. (2005). Anisotropic centroidal Voronoi tessellations and their applications. SIAM J. Sci. Comput., 26, 737-761.

Dupuis, F., Sadoc, J.-F., Jullien, R., Angelov, B., \& Mornon, J.-P. (2011). Voro3D: 3D Voronoi tessellations applied to protein structures. Bioinformatics, 21, 1715-1716.

Ferenc, J.-S., \& Néda, Z. (2007). On the size distribution of Poisson Voronoi cells. Phys. A , 385, 518-526.

Ferraro, M., \& Zaninetti, L. (2012). On the statistics of area size in two-dimensional thick Voronoi diagrams. Physica A Statistical Mechanics and its Applications, 391, 4575-4582.

González, D. L., \& Einstein, T. L. (2011). Voronoi cell patterns: Theoretical model and applications. Phys. Rev. E , 84, 051135 .

Heinrich, L., \& Schiile, E. (1995). Generation of the typical cell of a non-poissonian Johnson-Mehl tessellation. Communications in Statistics. Stochastic Models, 11, 541-560.

Hinde, A. L., \& Miles, R. (1980). Monte Carlo estimates of the distributions of the random polygons of the Voronoi tessellation with respect to a Poisson process. J. Stat. Comput. Simul., 10, 205-223.

Idrissi, A., Vyalov, I., Damay, P., Kiselev, M., Puhovski, Y., \& Jedlovszky, P. (2010). Local structure in sub- and supercritical CO2: A Voronoi polyhedra analysis study. Journal of Molecular Liquids, 153, 20-24.

Idrissi, A., Vyalov, I., Kiselev, M., Fedorov, M. V., \& Jedlovszky, P. (2011). Heterogeneity of the Local Structure in Sub-and Supercritical Ammonia: A Voronoi Polyhedra Analysis. The Journal of Physical Chemistry B, 115, 9646-9652.

Jedlovszky, P. (1999). Voronoi polyhedra analysis of the local structure of water from ambient to supercritical conditions. The Journal of chemical physics, 111, 5975-5985.

Jedlovszky, P. (2000). The local structure of various hydrogen bonded liquids: Voronoi polyhedra analysis of water, methanol, and HF. Journal of Chemical Physics , 113, 9113-9121.

Jedlovszky, P., Medvedev, N. N., \& Mezei, M. (2004). Effect of Cholesterol on the Properties of Phospholipid Membranes. 3. Local Lateral Structure. The Journal of Physical Chemistry B, 108, 465-472.

Kellerer, A. M. (1984). Chord-Length Distributions and Related Quantities for Spheroids. Radiation Research, 98, 425-437.

Khodabin, M., \& Ahmadabadi, A. (2010). Some properties of generalized gamma distribution. Math. Sci. Q. J., 4, 9-28.

Kiang, T. (1966). Random Fragmentation in Two and Three Dimensions. Z. Astrophys. , 64, 433-439.

Kolmogoroff, A. (1941). Confidence Limits for an Unknown Distribution Function. The Annals of Mathematical Statistics, 12, 461-463.

Lazar, E. A., Mason, J. K., MacPherson, R. D., \& Srolovitz, D. J. (2013). Statistical topology of three-dimensional Poisson-Voronoi cells and cell boundary networks. Phys. Rev. E, 88, 063309.

Le Caer, G., \& Ho, J. S. (1990). The Voronoi tessellation generated from eigenvalues of complex random matrices. Journal of Physics A: Mathematical and General, 23, 3279.

Massey, Frank J., J. (1951). The Kolmogorov-Smirnov Test for Goodness of Fit. Journal of the American Statistical Association, 46, 68-78.

Morokoff, W. J., \& Caflisch, R. E. (1994). Quasi-random sequences and their discrepancies. SIAM Journal on Scientific Computing, 15, 1251-1279.

Muche, L. (2010). Contact and chord length distribution functions of the Poisson-Voronoi tessellation in high dimensions. Adv. in Appl. Probab., 42, 48-68. 
Muche, L., \& Stoyan, D. (1992). Contact and Chord Length Distributions of the Poisson Voronoi Tessellation. Journal of Applied Probability, 29, 467-471.

Okabe, A., Boots, B., Sugihara, K.., \& Chiu, S. (2000). Spatial tessellations. Concepts and Applications of Voronoi diagrams, (2nd ed.). Chichester, New York: Wiley.

Poupon, A. (2004). Voronoi and Voronoi-related tessellations in studies of protein structure and interaction. Current Opinion in Structural Biology, 14, 233 - 241.

Press, W. H., Teukolsky, S. A., Vetterling, W. T.., \& Flannery, B. P. (1992). Numerical Recipes in FORTRAN. The Art of Scientific Computing. Cambridge, UK: Cambridge University Press.

Ruan, J. Z., Litt, M. H.., \& Krieger, I. M. (1988). Pore size distributions of foams from chord distributions of random lines: Mathematical inversion and computer simulation. Journal of Colloid and Interface Science, 126, $93-100$.

Smirnov, N. (1948). Table for Estimating the Goodness of Fit of Empirical Distributions. The Annals of Mathematical Statistics, 19, 279-281.

Sobol, I. M. (1967). The distribution of points in a cube and the approximate evaluation of integrals. USSR Comp. Math. Math. Phys., 7, 86-112.

Stoyan, D., Wagner, A., Hermann, H.., \& Elsner, A. (2011). Statistical characterization of the pore space of random systems of hard spheres. Journal of Non-Crystalline Solids, 357, 1508-1515.

Tanemura, M. (2003). Statistical Distributions of Poisson Voronoi Cells in Two and Three Dimensions. Forma, 18, 221-247.

Tanemura, M. (2005). Statistical distributions of the shape of Poisson Voronoi cells. In H. Syta (ed.), Voronoi's impact on modern science. Book III. Proceedings of the 3rd Voronoi conference on analytic number theory and spatial tessellations (pp. 193-202).

Tokita, N., Hirabayashi, M., Azuma, C.., \& Dotera, T. (2004). Voronoi space division of a polymer: Topological effects, free volume, and surface end segregation. The Journal of chemical physics, 120, 496-505.

Watson, G. S. (1971). Estimating functionals of particle size distributions. Biometrika, 58, 483-490.

\section{Copyrights}

Copyright for this article is retained by the author(s), with first publication rights granted to the journal.

This is an open-access article distributed under the terms and conditions of the Creative Commons Attribution license (http://creativecommons.org/licenses/by/3.0/). 